\title{
Solid phase extractive preconcentration coupled to gas chromatography-atomic emission detection for the determination of chlorophenols in water samples
}

\author{
Latif Elci ${ }^{*}$, Nina Kolbe, Sukru G. Elci ${ }^{1}$, Jan T. Anderson \\ Institute of Inorganic and Analytical Chemistry, University of Muenster, Corrensstrasse 30, 48149 Muenster, Germany
}

\section{A R T I C L E I N F O}

\section{Article history:}

Received 20 January 2011

Received in revised form 28 March 2011

Accepted 11 April 2011

Available online 17 April 2011

\section{Keywords:}

Chlorophenols

Derivatization

Ferrocenecarboxylic acid esters

Solid phase extraction

GC-AED

Water analysis

\begin{abstract}
A B S T R A C T
Solid-phase extraction (SPE) followed by derivatization and gas chromatography-atomic emission detection (GC-AED) was evaluated for the determination of five chlorophenols (CPs) in water samples. The derivatization was based on the esterification of phenolic compounds with ferrocenecarboxylic acid. The determination of the derivatized phenols was performed by GC-AED in the iron selective detection mode at $302 \mathrm{~nm}$. The described method was tested on spiked water samples.The overall method gave detection limits of 1.6-3.7 $\mathrm{ng} \mathrm{L}^{-1}$ and recoveries of $90.9-104.5 \%$ for the examined mono- to trichlorophenols in $10 \mathrm{~mL}$ water samples. The CPs extracted from a $10 \mathrm{~mL}$ water sample with SPE were concentrated into $100 \mu \mathrm{L}$ of organic solvent, a preconcentration factor of 100 . The method was applied to lake and tap water samples, and CP contents between 6 and $51 \mathrm{ng} \mathrm{L}^{-1}$ in lake water and between below the detection limit and $8 \mathrm{ng} \mathrm{L}^{-1}$ in tap water were found for different CPs. The method is quick, simple and gives excellent recoveries, limits of detection and standard deviations.
\end{abstract}

(C) 2011 Elsevier B.V. All rights reserved.

\section{Introduction}

Chlorinated phenols are widely used in the production of pesticides, herbicides, wood preservatives, antiseptic and disinfectants, as well as in the plastic, dye and pharmaceutical industries [1]. These compounds are thought to be highly toxic and lower chlorinated members have shown carcinogenic traits [2]. Owing to the toxicity of these compounds, both the US Environmental Protect Agency (EPA) [3] and the European Community (EC) [4] have included some phenols, mainly chlorophenols (CP) and nitrophenols, in their lists of priority pollutants. Four chlorophenols (2-CP, 2,4-DCP, 2,4,6-TCP, and PCP) as the most representative chlorophenols in water samples have been classified as priority pollutants by the U.S. Environmental Protection Agency [5]. The EC has mandated that the maximum admissible concentration of phenols in drinking water is $0.5 \mu \mathrm{g} \mathrm{L}^{-1}$ for the total content and $0.1 \mu \mathrm{g} \mathrm{L}^{-1}$ for individual ones [6].

Chlorophenols can be produced as a result of hydrolysis, oxidation and microbial degradation of chlorinated pesticides in the environment. They can enter water systems through various trans-

\footnotetext{
* Corresponding author. Permanent address: Pamukkale University, Art and Science Faculty Chemistry Department, Kınıklı, TR-20017 Denizli, Turkey, Tel.: +90 258 2963596, fax: +902582963535.

E-mail address: elci@pau.edu.tr (L. Elci).

1 İzmir Institute of Technology, Science Faculty, Chemistry Department, Urla, TR35430 Izmir, Turkey.
}

port mechanisms and can also chemisorb onto soil surfaces and aquatic sediments. Moreover, chlorine treatment of drinking water can generate CPs to create a major health threat [7]. Therefore, CPs are regarded as important enviromental pollutants and can frequently be found in surface water, tap water, industrial and domestic wastewaters $[8,9]$.

The need for monitoring and controlling the presence of these compounds in the aqueous environment is now well recognized, being essential for achieving good water-quality objectives. Many analytical methods, including capillary electrophoresis $[10,11]$, high-performance liquid chromatography (HPLC) [12] and gas chromatography [1] are available for the determination of CPs in water samples. Gas chromatography (GC), usually after derivatization, with flame-ionization detection (FID) [13,14], electron-capture detection (ECD) [15], mass spectrometric detection (MS) or microwave-induced plasma atomic emission spectrometry (MIP-AES) [16-19] is a common tool for the determination of phenols because of its high separation power and low limits of quantification.

Atomic emission detection (AED) is a selective and sensitive alternative to the use of other detectors for derivatized chlorinated phenols. It probably does not show any advantages for CPs if these are monitorind using the chlorine emission since this emission is not very strong and the selectivity vs. carbon is only ca. 27,000 [20]. The AED may be more interesting if an element with better detection characteristics than chlorine can be introduced into the chlorophenol through a derivatization reagent. An excellent choice is iron, which offers very low limits of detection (ca. $0.1 \mathrm{pg} / \mathrm{s}$ ) and 

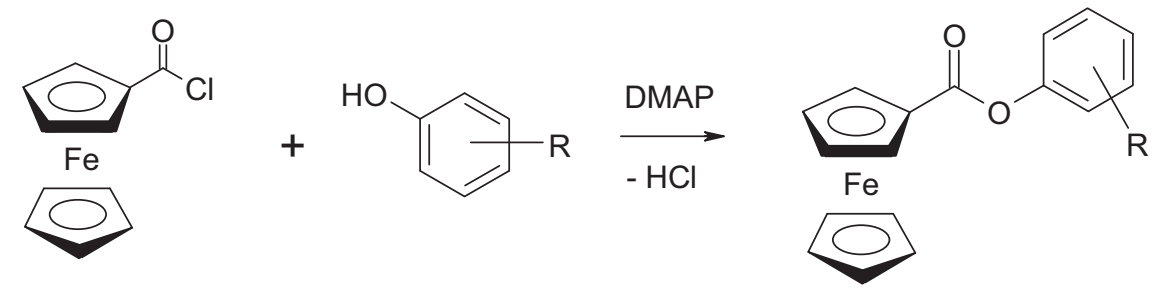

Fig. 1. The reaction of phenols with ferrocenecarboxylic acid chloride, introducing iron as the element to be detected by GC-AED.

extremely high selectivity vs. carbon (ca. 4.6 million) [20]. This element is easily introduced into phenols through their coupling with the acid chloride of ferrocenecarboxylic acid to yield the ferrocenecarboxylic acid esters (Fig. 1) [17-19].

In the reaction between ferrocenecarboxylic acid chloride (FCC) and a phenolic compound, each phenol is labelled with one iron atom. The method has been successfully applied to the determination of some phenolic compounds in non-aqueous materials like petroleum as well as for the determination of $o$-phenylphenol as a pesticide on citrus fruits $[17,18]$. To the best of our knowledge there has been no application of the method to the analysis of water samples. Due to the hydrolytic sensitivity of the acid chloride, phenols in water samples have to be transferred into a non-aqueous solvent before the derivatization step, thus necessitating an extraction procedure. Such procedures are commonly applied prior to GC analysis of CPs to extract and simultaneously to concentrate them to achieve the concentration levels required by legislation. Thus extensive liquid-liquid $[21,22]$ or solid-phase extraction (SPE) [5,23-25] methodologies followed by derivatization have been applied prior to GC analysis. Solid-phase extraction is the most frequently used technique for preconcentration of CPs and consequently we make use of an SPE column here as an ideal way of transferring the phenolic compounds from water samples into an organic solvent.

Recently, several studies have shown that phenols, including CPs, can be extracted using SPE based on polystyrenedivinylbenzene (PS-DVB) with good recoveries and detection limits at the sub-mg/L level $[26,27]$. PS-DVB resins have greater analyte retention, mainly for polar compounds, than bonded silicas because their hydrophobic surface contains a large number of active aromatic sites, which allow $\pi-\pi$ interactions with unsaturated analytes [28]. It is well known that the retention of phenols on PS-DVB resins is the result of a reversed-phase mechanism. As a result, here Amberchrom 161C, a PS-DVB resin, is explored as the solid phase material for SPE because of its large surface area and small particles.

This paper reports a method for the determination of 2-chlorophenol (2-CP), 3-chlorophenol (3-CP), 4-chlorophenol (4-CP), 2,4-dichlorophenol (2,4-DCP) and 2,4,6-trichlorophenol $(2,4,6-\mathrm{TCP})$ in water samples, therefore including the EU and EPA priority pollutants 2-CP, 2,4-DCP and 2,4,6-TCP. The method involves a solid phase extraction procedure using an Amberchrom 161C resin column, then a derivatization of the CPs with ferrocenecarboxylic acid chloride followed by their determination with GC-AED. The proposed method was successfully applied to the determination of CPs in lake and tap water samples.

\section{Experimental}

\subsection{Material and chemicals}

2-Chlorophenol, 3-chlorophenol, 4-chlorophenol (98\% w/w purity), 2,4-dichlorophenol and 2,4,6-trichlorophenol standards of 99\% purity were obtained from commercial suppliers (Aldrich, Fluka, Merck and Riedel-de Haën). A stock solution, which is a mixture of the chlorophenols at $100 \mathrm{mM}$ was prepared in toluene, diluted to the required concentration to produce working solutions, and then stored in the dark at $4{ }^{\circ} \mathrm{C}$ in a refrigerator. The used water was prepared with a Milli-Q water purification system (Millipore, Bedford, MA).

4-fluoro-2-methylphenol ferrocenecarboxylic acid ester (4F2MPE) and 4-fluoro-2-methylphenol (4F2MP), both purchased from Sigma-Aldrich, Germany, were used as quantification internal standards for esterification, and the combination of the SPE and derivatization, respectively. Toluene, cyclohexane, and dichloromethane (all pesticide grade, Fluka) were purified by percolation through aluminum oxide before use. Further chemicals used were anhydrous sodium sulfate (99\%, Fluka) and 4-(dimethylamino)pyridine (DMAP, 99\%, Acros). Aluminum oxide (chromatography grade, neutral, Fluka) was activated at $450{ }^{\circ} \mathrm{C}$, and stored at $160^{\circ} \mathrm{C}$ for at least $24 \mathrm{~h}$ for a water content of $1.2 \%$. The derivatization reagent ferrocenecarboxylic acid chloride (FCC) was synthesized from ferrocene carboxylic acid with oxalyl chloride, following the published procedure $[17,19]$. While the deeply red product is stable for almost one year if stored at $-18^{\circ} \mathrm{C}$ in the dark under argon gas, solutions in dichloromethane should be prepared freshly just prior to use.

The Amberchrom $161 \mathrm{C}$ resin was supplied by Supelco. It consists of a polystyrene-divinylbenzene copolymer (PS-DVB), contains a hydrophobic structure and is a spherical resin with an average particle size of $50-100 \mu \mathrm{m}$, an average pore size of $150 \AA$ and a surface area of $720 \mathrm{~m}^{2} / \mathrm{g}$ [25]. Empty SPE glass columns with PTFE frits were purchased from Merck (Darmstadt, Germany).

\subsection{Apparatus}

The Agilent GC-AED system consists of a 6890 N GC and a G2350A AED, equipped with a $30 \mathrm{~m} \times 0.25 \mathrm{~mm}$ i.d. $\times 0.25 \mu \mathrm{m}$ film thickness DB-5ms column (J\&W Scientific, Folsom, CA, USA), a Gerstel MPS2 autosampler, and a Gerstel CIS-injector (Gerstel, Mülheim a.d.R., Germany). The instrumental parameters were taken from Ref. [18]. The oven temperature was programmed as follows: $60^{\circ} \mathrm{C}$ starting temperature, kept for $0.5 \mathrm{~min}$, temperature ramp at $45^{\circ} \mathrm{C} / \mathrm{min}$ to $295^{\circ} \mathrm{C}$, then at $2^{\circ} \mathrm{C} / \mathrm{min}$ to $300^{\circ} \mathrm{C}$, kept for $3 \mathrm{~min}$. Other GC-AED conditions were: injector initial temperature, $60^{\circ} \mathrm{C}$, heated at $12^{\circ} \mathrm{C} / \mathrm{s}$ to $300^{\circ} \mathrm{C}$; helium carrier gas with $40 \mathrm{~cm} / \mathrm{s}$ constant velocity; transfer line and cavity temperature $300^{\circ} \mathrm{C}$. Helium make-up flow for the AED is $240 \mathrm{~mL} / \mathrm{min}$; hydrogen and oxygen plasma pressures are 15 and 20 psi, respectively.

\subsection{Sampling}

The water from the Lake Aasee in Münster was collected daily in glass sampling bottles which were chemically cleaned using acetone and dichloromethane succesively before the collection. It was taken from a depth of $10 \mathrm{~cm}$ and was filtered through a glass-fiber filter $(0.45 \mu \mathrm{m}$ pore size $)$ to eliminate particulate matter before the preconcentration step. The samples were analyzed on the same day to avoid any microbial degradation of analytes. Tap water samples were taken directly from the tap in a laboratory at the University of 
Münster. The $\mathrm{pH}$ of the tap water and the lake water samples was measured as 6.0 and 6.5 , respectively.

\subsection{Solid phase extraction cartridges}

Amberchrom 161C resin packed cartridges were prepared from empty SPE glass cartridges ( $3 \mathrm{~mL}$ ). $0.5 \mathrm{~g}$ of Amberchrom $161 \mathrm{C}$ resin was packed into the cartridge after a PTFE frit had been placed at the cartridge bottom. Another PTFE frit was introduced at the top of the cartridge to fix the height of the resin. The dry resin bed height in the column was approximately $2.5 \mathrm{~cm}$. It was washed successively with DCM and twice distilled water. The column could be reused at least ten times for the preconcentration of CPs from water samples.

To prepare the mini aluminum oxide column, ca. $1.7 \mathrm{~g}$ of aluminum oxide was packed into a $3 \mathrm{~mL}$ SPE glass cartridge under DCM. The column was used to separate ferrocenecarboxylic acid esters from the excess of FCC and DMAP in the derivatization solution [19]. These columns were used only once.

\subsection{Esterification of chlorophenos with ferrocenecarboxylic acid chloride}

The esterification with FCC has been used previously for the determination of alkylated phenols [17-19]. Since CPs have not been investigated in this procedure previously, their derivatization was first evaluated by determination of spiked toluene solutions. To $5 \mathrm{~mL}$ of toluene was added $50 \mu \mathrm{L}$ of a $100 \mu \mathrm{M}$ mixed solution of 2-CP, 3-CP, 4-CP, 2,4-DCP and 2,4,6-TCP, and $50 \mu \mathrm{L}$ of a $100 \mu \mathrm{M}$ ferrocenecarboxylic acid ester of 4-fluoro-2-methylphenol

(4F2MPE) in toluene as a quantification internal standard. The derivatives are stable for several months at $5^{\circ} \mathrm{C}$. The known esterification procedure [18] was used as detailed below.

\subsection{Preconcentration and derivatization for water analysis}

A $10 \mathrm{~mL}$ portion of the water samples collected either from the Lake Aasee in Münster or from a tap at the University of Münster was passed through the SPE column packed with Amberchrom $161 \mathrm{C}$ resin at a flow rate of $2 \mathrm{~mL} / \mathrm{min}$. The residue of water in the resin was removed by attaching the column to a pump and lowering the pressure, and then the resin was dried by introducing a gentle flow of argon gas at the column head for $25-30 \mathrm{~min}$. Finally, the retained chlorophenols were recovered by eluting the column with $5.0 \mathrm{~mL}$ of dichloromethane at a flow rate of $1 \mathrm{~mL} / \mathrm{min}$ into a $10 \mathrm{~mL}$ vial containing ca. $1.0 \mathrm{~g}$ of anhydrous sodium sulfate to remove any residual water from the solution. 4-Fluoro-2-methylphenol as internal standard was added to the vial. To recondition the column for the next sample, it was washed once with $5.0 \mathrm{~mL}$ of DCM and then twice with $5.0 \mathrm{~mL}$ of twice distilled water.

Derivatization of the preconcentrated CPs and 4F2MP [17-19]: $20 \mathrm{mg}$ FCC as derivatization agent and $20 \mathrm{mg}$ DMAP as catalyst were added to the vial. Because of the probable residual water in the effluent we added more than $15 \mathrm{mg}$ of FCC and DMAP. After a reaction time of $10 \mathrm{~min}$ at room temperature, the solution was added to the top of the aluminum oxide column and the ferrocenecarboxylic acid esters of the CPs were eluted with $5.0 \mathrm{~mL}$ of dichloromethane (the DMAP and the excess FCC remained on the column). The derivatized sample in the vial was further concentrated almost to dryness using a gentle flow of nitrogen at $40^{\circ} \mathrm{C}$. The remaining material was dissolved in $100 \mu \mathrm{L}$ of cyclohexane for analysis by GC-AED.

\section{Results and discussion}

The ferrocenecarboxylic acid derivatization provides a very sensitive and selective method to analyze CPs as ferrocenecarboxylic acid esters (FE) based on GC-AED in the iron selective mode [20]. The conventional derivatization procedures (acetylation, silylation) are less sensitive and selective method than the derivatization with ferrocenecarboxylic acid, for AED. Because, an element (e.g. chlorine) which is less sensitive than iron, is used as analyzed element in the combination of conventional derivatizations with GC-AED. The advantage of using this new derivatization procedure is high selectivity and sensitivity. This derivatization reaction has previously been used for alkyl- and phenyl substituted but not for chlorinated phenols. Since the electronic situation in these different kinds of phenols is completely different, it was necessary to investigate the optimum conditions for the coupling of the CPs with ferrocenecarboxylic acid chloride.

\subsection{Optimization of the derivatization with ferrocenecarboxylic acid chloride}

Variables such as the amount of FFC (derivatizing reagent) and of DMAP (catalyst), derivatization time and effect of microwave energy were studied for the derivatization of the CPs. For this, each variable is changed in turn, while the others are held constant, until a maximum derivatization yield (\%) has been found. 4F2MPE was used as an internal standard.

The effect of FCC and DMAP amounts at 10 min reaction time was investigated first at a constant amount of DMAP $(10 \mathrm{mg})$ and a constant amount of FCC $(15 \mathrm{mg})$, respectively. The best yields were obtained with $15 \mathrm{mg}$ of FCC and $15 \mathrm{mg}$ of DMAP, which were the largest amounts tested in these experiments. The derivatization yields for 2-CP, 3-CP, 4-CP, 2,4-DCP and 2,4,6-TCP were found to be $92.5,91.6,91.2,81.6$ and $83.6 \%$, respectively. Obviously the esterification yield of CPs depends on the amount of both FFC and DMAP.

We also tested the effect of reaction time on the yield. The yields hardly varied for reaction times between 5 and $15 \mathrm{~min}$ at constant amounts of FCC (15 mg) and DMAP ( $10 \mathrm{mg}$ ). Thus it seemed advisable to work with at least $15 \mathrm{mg}$ of DMAP, $15 \mathrm{mg}$ FFC and a reaction time of $10 \mathrm{~min}$. Finally the effect of microwaves on the reaction yield was tested. The derivatization solution was exposed to microwaves for $2 \mathrm{~min}$. The derivatization yields for 2-CP, 3-CP, 4-CP, 2,4-DCP and $2,4,6$-TCP were found to be $90.3,93.4,87.5,90.6$ and $88.9 \%$, respectively, for an average yield of $90.1 \%$ vs. $88.1 \%$ without microwaves above. Microwaves therefore do not seem to improve the yield and this technique was not used any further.

\subsection{Optimization of the solid phase extraction}

The optimization of the complete method - the combination of SPE and derivatization - was first evaluated by determination of the standard CPs in $50 \mu \mathrm{L}$ of a $100 \mu \mathrm{M}$ solution spiked into $10 \mathrm{~mL}$ of distilled water. The water was also spiked with $50 \mu \mathrm{L}$ of a $100 \mu \mathrm{M}$ solution of 4-fluoro-2-methylphenol (4F2MP) as the quantification internal standard. During the optimization of the SPE step, the amounts of FCC and DMAP were ca. $20 \mathrm{mg}$, more than their optimum amounts given above because of probable residual water or moisture in the effluent from the SPE that might decompose the acid chloride.

The $\mathrm{pH}$ of the sample solution is generally accepted as the key parameter for a quantitative extraction of phenols. We chose $\mathrm{pH}$ 2 and 6 as test pHs based on $\mathrm{pK}_{\mathrm{a}}$ values of the examined CPs. The recoveries of the CPs were obtained with the model solution containing a mixture of five CPs in distilled water. The complete procedure was applied. As indicated in Table 1, the recovery values of the CPs for $\mathrm{pH} 2$ and 6 ranged from 83.5 to $104.2 \%$ and from 84.3 to $99.4 \%$, respectively. There was no significant dependance of the recoveries on the $\mathrm{pH}$ in the range 2.0-6.0 with the exception of 2,4,6-TCP. An increase in $\mathrm{pH}$ produced a decrease in recovery of 
Table 1

Effect of sample pH on the SPE recovery (\%) of the CPs.

\begin{tabular}{llcl}
\hline \multirow{2}{*}{ Compounds } & $\mathrm{pK}_{\mathrm{a}}^{\mathrm{a}}$ & \multicolumn{2}{c}{ Recovery, \% } \\
\cline { 3 - 4 } & & $\mathrm{pH} 2$ & $\mathrm{pH} \mathrm{6}$ \\
\hline $2-\mathrm{CP}$ & 8.52 & 104.2 & 99.4 \\
$3-\mathrm{CP}$ & 9.37 & 96.7 & 98.8 \\
$4-\mathrm{CP}$ & 8.97 & 101.7 & 93.6 \\
$2,4-\mathrm{DCP}$ & 6.80 & 83.5 & 84.3 \\
$2,4,6-\mathrm{TCP}$ & 5.80 & 102.7 & 91.6 \\
\hline
\end{tabular}

a from [25].

Table 2

Analytical data for the determined chlorophenols.

\begin{tabular}{lllll}
\hline CPs & LOD $\left(\mathrm{ng} \mathrm{L}^{-1}\right)$ & LOQ $\left(\mathrm{ng} \mathrm{L}^{-1}\right)$ & Recovery $(\%)(n=6)$ & RSD $(\%)(n=6)$ \\
\hline 2-CP & 1.6 & 4.3 & 104.5 & 4.1 \\
3-CP & 2.4 & 6.7 & 103.2 & 9.4 \\
$4-\mathrm{CP}$ & 3.7 & 9.9 & 90.9 & 12.0 \\
2,4-DCP & 3.4 & 8.5 & 93.6 & 14.8 \\
2,4,6-TCP & 3.1 & 7.5 & 101.1 & 11.8 \\
\hline
\end{tabular}

a Detection limits are calculated with a signal-to-noise ratio of 3.

b Quantification limits are calculated with a signal-to-noise ratio of 10 .

this compound, probably because of its higher acidity compared to the other CPs.

There is no significant difference in recovery for sample volumes between $2 \mathrm{~mL}$ and $10 \mathrm{~mL}$ at a flow rate of $2 \mathrm{~mL} / \mathrm{min}$. The effect of sample volume was not examined for larger volumes than $10 \mathrm{~mL}$. In such cases the preconcentration factor was 100 .

\subsection{Analytical performance}

The following figures of merit were investigated for the full process under the optimal experimental conditions: limit of detection (LOD), precision and accuracy. The LODs for the chromatography step, based on peaks of a signal-to-noise ratio of 3, ranged from $1.6 \mathrm{ng} \mathrm{L}^{-1}$ to $3.7 \mathrm{ng} \mathrm{L}^{-1}$ (Table 2). The limits of quantification (LOQ) for the CPs were calculated using a signal-to-noise ratio of 10 (Table 2). Of note is the fact that the LOQs obtained were better than those obtained with both solvent extraction in EPA methods 604 (GC-FID) and 625 (GC-MS) [21,29]. The LOQs for 2-CP, 4-CP and 2,4DCP were better than those obtained using HS-SPME-GC-MS but not for 2,4,6-TCP $\left(2 \mathrm{ng} \mathrm{L}^{-1}\right)$ [30]. The LOQs obtained in this work are lower by a factor of 10-100 than those obtained with a purge-andtrap preconcentration coupled to a GC-AED $\left(510 \mathrm{ng} \mathrm{L}^{-1}\right.$ for $2-\mathrm{CP}$, $300 \mathrm{ng} \mathrm{L}^{-1}$ for 2,4-DCP and $77 \mathrm{ng} \mathrm{L}^{-1} 2,4,6$-TCP) [16]. This is probably due to the use of the more sensitive iron line here rather than the chlorine line at $479 \mathrm{~nm}$. Purge-assisted headspace SPME combined with GC-MS gave lower LODs for some CPs $\left(1.4 \mathrm{ng} \mathrm{L}^{-1}\right.$ for DCP and $1.1 \mathrm{ng} \mathrm{L}^{-1}$ for $\left.2,4,6-\mathrm{TCP}\right)$ than the present GC-AED determination [1].

The precision of the procedure (relative standard deviation, RSD) was calculated. Six replicate determinations of CPs added as $20 \mu \mathrm{L}$ of a $100 \mu \mathrm{M}$ solution to $10 \mathrm{~mL}$ of tap water indicated that the RSDs range from 4.1 to $14.8 \%$ and the recoveries from 90.9 to $104.5 \%$, both showing comparable values at this concentration level with the values given in the references mentioned above $[1,16,30]$.

To validate the accuracy of the complete method, the recoveries were evaluated for each compound in spiked water $(10 \mathrm{~mL})$ with varying amounts of the standard solution $(15,25$ and $40 \mu \mathrm{L}$ of a $100 \mu \mathrm{M}$ solution of the CPs). As indicated in Table 3, the recovery values of the CPs spiked in tap water and lake water samples ranged from 82.3 to $113.7 \%$ and from 79.9 to $112.2 \%$, respectively. These results demonstrate that the matrixes of the analyzed real-world water samples have no effect on this method.

\subsection{Analysis of real water samples}

After the analytical performance was found to be satisfactory, the next step was to determine how well the method works when applied to the analysis of CPs in lake and tap waters. The determined CP concentrations are presented in Table 4 . As expected, the concentrations of the CPs in lake water were higher than those in the tap water. The chlorophenol contents in all water sample are lower than the maximum admissible concentration of phenols in drinking water by the EC legislations [6]. Four replicate determinations of the CPs present in $10 \mathrm{~mL}$ sample gave satisfactory RSD values, ranging from 5 to $16 \%$.

In Fig. 2 is depicted the gas chromatogram with atomic emission detection of the lake water sample after derivatization with FCC. It can be seen that even at this low level of CPs (6-50 ng/L), an excellent signal-to-noise ratio is obtained. A few unidentified peaks are likely to be derived from other phenols from the water, such as phenol itself (eluting immediately after 2FP) and o-cresol (immediately following 4F2MP). The large peak immediately before that of 2FP is derived from a sideproduct of the derivatization and is thus

Table 3

Recoveries of CPs from spiked tap and lake water samples (10 mL).

\begin{tabular}{|c|c|c|c|c|c|c|c|}
\hline \multirow[t]{2}{*}{ CPs } & \multirow[t]{2}{*}{ Added vol. ${ }^{\mathrm{a}}(\mu \mathrm{L})$} & \multicolumn{3}{|c|}{ Tap water ( $n=3$ samples) } & \multicolumn{3}{|c|}{ Lake water ( $n=3$ samples) } \\
\hline & & Added (ng L $\left.{ }^{-1}\right)$ & Found $^{\mathrm{b}}\left(\mathrm{ng} \mathrm{L}^{-1}\right)$ & Recovery (\%) & Added $\left(\operatorname{ng~L}^{-1}\right)$ & Found $^{\mathrm{b}}\left(\mathrm{ng} \mathrm{L}^{-1}\right)$ & Recovery (\%) \\
\hline $2-\mathrm{CP}$ & 15 & 28.8 & 31.4 & 109.0 & - & - & - \\
\hline 3-CP & & 28.8 & 34.8 & 93.1 & - & - & - \\
\hline 4-CP & & 28.8 & 29.7 & 85.8 & - & - & - \\
\hline 2,4-DCP & & 36.5 & 30.0 & 82.3 & - & - & - \\
\hline $2,4,6-\mathrm{TCP}$ & & 44.3 & 49.5 & 111.7 & - & - & - \\
\hline $2-\mathrm{CP}$ & 25 & 48.0 & 50.0 & 104.2 & 48.0 & 49.2 & 102.4 \\
\hline $3-\mathrm{CP}$ & & 48.0 & 60.6 & 109.7 & 48.0 & 46.2 & 96.3 \\
\hline 4-CP & & 48.0 & 48.3 & 90.2 & 48.0 & 53.8 & 112.2 \\
\hline 2,4-DCP & & 60.8 & 62.7 & 103.2 & 60.8 & 51.6 & 84.9 \\
\hline 2,4,6-ТCP & & 73.8 & 72.2 & 97.9 & 73.8 & 58.9 & 79.9 \\
\hline $2-\mathrm{CP}$ & 40 & 76.8 & 82.8 & 107.8 & 76.8 & 76.0 & 99.0 \\
\hline $3-C P$ & & 76.8 & 95.3 & 113.7 & 76.8 & 74.4 & 96.9 \\
\hline 4-CP & & 76.8 & 88.7 & 109.0 & 76.8 & 73.1 & 95.2 \\
\hline 2,4-DCP & & 97.3 & 108.8 & 111.8 & 97.3 & 95.7 & 98.4 \\
\hline $2,4,6-\mathrm{TCP}$ & & 118.1 & 119.3 & 101.0 & 118.1 & 106.1 & 89.8 \\
\hline
\end{tabular}

a Added volume from the mixed solution of CPs as $100 \mu \mathrm{M}$.

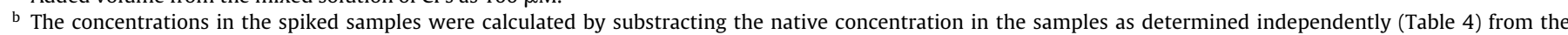
measured total concentrations. 


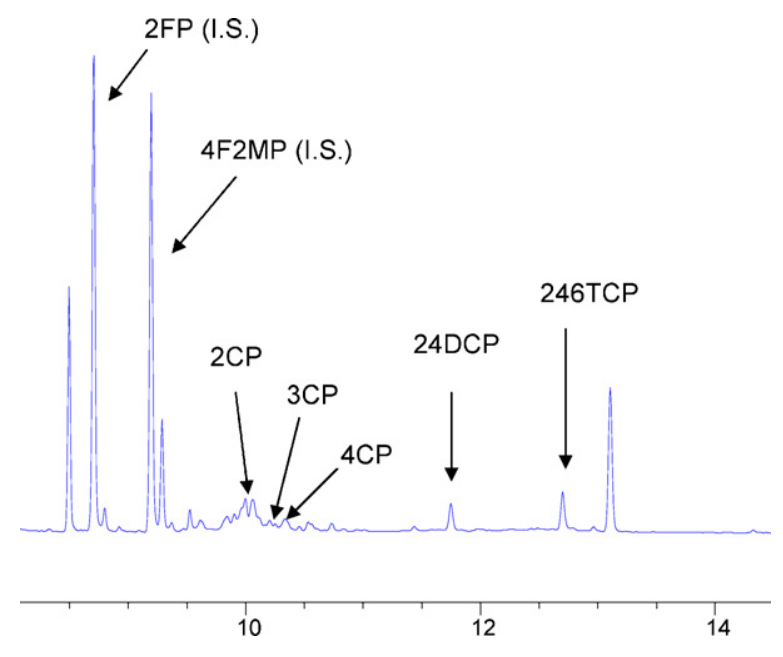

Fig. 2. A gas chromatogram with atomic emission detection of the lake water sample with the identified chlorophenols indicated. 2FP and 4F2MP are the internal standards (I.S.) 2-fluorophenol and 4-fluoro-2-methylphenol.

Table 4

Concentration of the CPs in lake and tap water samples.

\begin{tabular}{lll}
\hline Analytes & $\begin{array}{l}\text { Tap water, mean } \pm \mathrm{s} \\
\left(\mathrm{ng} \mathrm{L}^{-1}\right), n=4 \text { samples }\end{array}$ & $\begin{array}{l}\text { Lake water, mean } \pm \mathrm{s} \\
\left(\mathrm{ng} \mathrm{L}^{-1}\right), n=4 \text { samples }\end{array}$ \\
\hline $2-\mathrm{CP}$ & $\mathrm{ND}^{\mathrm{a}}$ & $18 \pm 3$ \\
$3-\mathrm{CP}$ & $8 \pm 1$ & $6 \pm 1$ \\
$4-\mathrm{CP}$ & $5 \pm 1$ & $15 \pm 1$ \\
$2,4-\mathrm{DCP}$ & $\mathrm{ND}$ & $34 \pm 3$ \\
$2,4,6-\mathrm{TCP}$ & $\mathrm{ND}$ & $51 \pm 5$ \\
\hline
\end{tabular}

a ND indicates below the limit of detection.

not related to the sample. An unknown substance elutes very close to the peak for $2 \mathrm{CP}$ so that the integration of this peak area is not very reliable.

\section{Conclusions}

The results show that the SPE-derivatization method combined with GC-AED is successful for the determination of trace amounts of CPs in water samples. A $10 \mathrm{~mL}$ sample can be extracted with SPE and preconcentrated by a factor of 100 to give detection limits of $8.2-18.7 \mathrm{ng} \mathrm{L}^{-1}$, about ten times lower than the maximum permissible levels in current legislation. A further reduction of the detection limits can be achieved by increasing the sample volume processed on SPE before the derivatization. The recoveries of CPs in the water samples exceed $90 \%$ with RSDs of less than $12 \%$ (except for 2-CP in lake water but this may be due to the coelution evident in Fig. 2 and the concomitant difficulty of identifying the peak inte- gration limits). Based on the simplicity, low quantification limits, accuracy and repeatability, this method can be recommended to determine CPs in natural water samples such as river water, lake water, drinking water, waste water and ground water, etc.

The derivatization procedure is very rugged, rapid, and easy to perform. It may concluded from almost quantitative derivatization yields that analyte losses are minimal during sample preparation and storage. Furthermore it is easily connected to a solid phase extraction of CPs.

\section{Acknowledgement}

L. Elçi is grateful to the Deutscher Akademischer Austauschdienst (DAAD) for financial support of this study.

\section{References}

[1] H.P. Ho, R.J. Lee, M.R. Lee, J. Chromatogr. A 1213 (2008) 245.

[2] K. Kawamoto, K. Urano, Chemosphere 18 (1989) 1987.

[3] Sample and Analysis Procedure for Screening of Industrial Effluents for Priority Pollutants, US Environmental Protection Agency, Environment Monitoring and Support Laboratory, Cincinnati, OH, 1977.

[4] The list of priority substances in the field of water policy and amending directive, Council directive 2455/2001/ECC, Official Journal L331, November 20, 2001, pp. 1-5.

[5] L.E. Sojo, J. Djauhari, J. Chromatogr. A 840 (1999) 21

[6] Drinking Water Directive 80/778/EEC, Commission of the European Communities, Brussels, 1975.

[7] R.C.C. Wegman, A.W.M. Hofstee, Water Res. 13 (1979) 651.

[8] M.N. Sarrion, F.J. Santos, M.T. Galceran, J. Chromatogr. A 947 (2002) 155.

[9] I. Rodríguez, M.I. Turnes, M.H. Bollaín, M.C. Mejuto, R. Cela, J. Chromatogr. A 778 (1997) 279.

[10] I. Ali, H.Y. Aboul-Enein, Fresen. Environ. Bull. 11 (2002) 36.

[11] P. Puig, F. Borrull, M. Calull, C. Aguilar, Anal. Chim. Acta 616 (2008) 1.

[12] G. Lamprecht, J. Huber, J. Chromatogr. A 667 (1994) 47.

[13] H. Faraji, J. Chromatogr. A 1087 (2005) 283.

[14] A. Kovács, A. Kende, M. Mörtl, G. Volk, T. Rikker, K. Torkos, J. Chromatogr. A 1194 (2008) 139.

[15] H. Bagheri, M. Saraji, J. Chromatogr. A 910 (2001) 87.

[16] N.Campillo, N. Aguinaga, P. Viñas, I. López-García, M. Hernández-Córdoba, Anal. Chim. Acta 552 (2005) 182.

[17] J. Rolfes, J.T. Andersson, Anal. Commun. 33 (1996) 429.

[18] N. Kolbe, J.T. Andersson, J. Agric. Food Chem. 54 (2006) 5736.

[19] J. Rolfes, J.T. Andersson, Anal. Chem. 73 (2001) 3073.

[20] J.T. Andersson, Anal. Bioanal. Chem. 373 (2002) 344

[21] Environmental Protection Agency (EPA), EPA Method 625, Base/Neutral and Acids, Part VIII, 40 CFR Part 136, EPA, Washington, DC, USA, 26 October 1984, p. 153.

[22] N. Fattahi, Y. Assadi, M.R.M. Hosseini, E.Z. Jahromi, J. Chromatogr. A 1157 (2007) 23.

[23] R. Wissiack, E. Rosenberg, M. Grasserbauer, J. Chromatogr. A 896 (2001) 159.

[24] A. Martínez-Uruñuela, I. Rodríguez, R. Cela, J.M. González-Sáiz, C. Pizarro, Anal. Chim. Acta 549 (2005) 117.

[25] I. Rodríguez, M.P. Llompart, R. Cela, J. Chromatogr. A 885 (2000) 291.

[26] M.C. Quintana, L. Ramos, Trends Anal. Chem. 27 (2008) 418.

[27] M. Jin, X. Chen, J. Liq. Chromatogr. Rel. Technol. 29 (2006) 1369.

[28] N. Masqué, M. Galià, R.M. Marcé, F. Borrull, J. Chromatogr. A 803 (1998) 147.

[29] Fed. Reg., EPA method 604, Phenols, Part VIII, 40 CFR Part 136, US Environmental Protection Agency, Washington, D.C., 26 October 1984, p. 58.

[30] M. Llompart, M. Lourido, P. Landín, C. García-Jares, R. Cela, J. Chromatogr. A 963 (2002) 137. 